\title{
Is maternal education level associated with diet in 10-year-old children?
}

\author{
Victoria L Cribb ${ }^{1}$, Louise R Jones ${ }^{1}$, Imogen S Rogers ${ }^{2}$, Andrew R Ness ${ }^{3}$ and \\ Pauline M Emmett ${ }^{1, *}+$ \\ ${ }^{1}$ School of Social and Community Medicine, University of Bristol, Bristol, UK: ${ }^{2}$ School of Pharmacy and \\ Biomolecular Sciences, University of Brighton, Brighton, UK: ${ }^{3}$ Department of Oral and Dental Science, Bristol \\ Dental School, University of Bristol, Bristol, UK
}

Submitted 20 April 2010: Accepted 24 January 2011: First published online 18 March 2011

\begin{abstract}
Objective: To examine the associations between maternal education level and diet in 10-year-old children.

Design: Three-day diet diaries (child completed with parental help) were collected. Height and weight were measured in research clinics. Maternal education level was derived from a questionnaire completed during pregnancy and classified into low, medium or high. One-way ANOVA was undertaken to compare maternal education groups for nutrient intakes and the Kruskal-Wallis test used for food consumption.

Setting: Avon Longitudinal Study of Parents and Children (ALSPAC), Bristol, UK. Subjects: Children ( $n$ 7474) who provided dietary data at age 10 years.

Results: A large proportion (60\%) of the sample was classified as plausible reporters, with under-reporting accounting for 36\%. No clear differences were found for intakes of energy or macronutrients between maternal education groups for plausible reporters. However, there were marked differences in micronutrient intakes especially for vitamin $\mathrm{C}$, retinol equivalents and folate, highlighting lower diet quality with lower maternal education level. Intakes of fruit and vegetables showed a positive gradient with increasing maternal education $(57 \% v .79 \%$ consumed fresh fruit in low and high educational groups, respectively). A trend towards higher intake in the lower educated group was shown for less healthy foods (meat pies $P<0 \cdot 001$; sausages, burgers and kebabs $P<0 \cdot 001$ ).

Conclusions: The quality of children's diet at 10 years was related to maternal education level. Lower maternal education was associated with less healthy food choices that could be detrimental to health. Further research is needed to establish if these associations can be explained by other socio-economic factors.
\end{abstract}

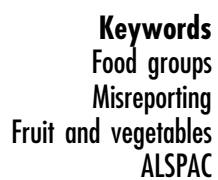

ALSPAC
Diet is important in the health and development of children, and can impact on later health outcomes ${ }^{(1)}$. The early adoption of healthy lifestyle behaviours can reduce the risk of disease ${ }^{(2,3)}$ and have implications for the child's behaviour and school performance ${ }^{(4,5)}$. Establishing and maintaining healthy eating habits is important because habits formed in early life are likely to continue into adulthood $^{(2,4)}$, therefore it is very important to understand influences on children's diets. It has been suggested that maternal education may play a key role in the quality of children's diets ${ }^{(6,7)}$.

\footnotetext{
† Correspondence address: Centre for Child \& Adolescent Health, Schoo of Social and Community Medicine, Oakfield House, Oakfield Grove, Bristol BS8 2BN, UK.
}

Parents, especially mothers, help children learn and develop both eating habits and food choices $^{(8)}$; this may act through their personal preferences ${ }^{(9,10)}$, attitudes to food $^{(11)}$ and their knowledge and understanding of the benefits of a healthy $\operatorname{diet}^{(9,12)}$. However, children can exercise their own control independent of their parents; it has been observed that children varied the foods they consumed depending on whether or not they were being observed by their parents ${ }^{(13)}$

Several studies have examined the relationship between maternal education and diet in infants and children. Higher maternal educational status was associated with longer duration of breast-feeding, improved physical growth, higher intakes of micronutrients, fruits and vegetables, and lower intake of soft drinks ${ }^{(6,7,14-16)}$. A relatively small 
study ( $n$ 133) showed maternal education to be a strong determinant for better nutritional intake in primaryschool children aged $7-10$ years ${ }^{(14)}$. Studies have found similar links between educational status and diet in adults; a meta-analysis of studies from seven European countries reported a less healthy dietary pattern in the least educated groups ${ }^{(17)}$, while among Chinese Americans intakes of grains, fruits and vegetables rose with education and income ${ }^{(18)}$.

Despite the evidence from these studies, limited research is available examining the extent to which maternal education level is associated with dietary intake in older children. Therefore, the aim of the present study was to use data from a well-characterised cohort of children followed from birth to establish whether the educational background of the mother is associated with dietary habits in 10-year-old children.

\section{Methods}

\section{Subjects}

Subjects were children participating in the Avon Longitudinal Study of Parents and Children (ALSPAC), an ongoing longitudinal cohort study designed to investigate the health and development of children. The study design has been described in more detail elsewhere ${ }^{(19)}$ (see also http:// www.bristol.ac.uk/alspac/). Briefly, pregnant women were eligible if they had an expected delivery date between April 1991 and December 1992 and were resident in the former Avon Health Authority in South West England. This established a cohort of 14541 resulting in 13988 children (alive at 12 months) with 548 new subjects recruited at age 7 years (total children 14536). The primary source of data collected was via parental self-completion questionnaires. At recruitment the ALSPAC cohort was compared with the 1991 National Census data for mothers with infants aged $<1$ year resident in the area; they were similar except for a slight shortfall in those living in rented accommodation, singleparent families and ethnic minorities. Ethical approval for the study was obtained from the ALSPAC Law and Ethics Committee and the Local Research Ethic Committees.

\section{Dietary assessment}

Dietary data were collected between February 2002 and October 2003, when each child (mean age $10 \cdot 6$ years) and their main carer were invited to attend a research clinic. Prior to the visit, the children were asked to record in a structured diary (using household measures) all food and drink consumed over three individual days, two weekdays and one weekend day (self-selected and not necessarily consecutive), with the help of their carer. A full description of the food and drink consumed was requested with a description of any leftovers. They were asked to bring completed diaries to the clinic, where they were interviewed by a nutrition fieldworker to expand the description; for example, gaining further information on portion size, cooking methods and any food/drink missed out. If no diaries had been brought to the clinic, the fieldworker conducted a $24 \mathrm{~h}$ recall during which the child was asked about everything consumed on the previous day. A short questionnaire was also included, asking about the use of vitamin supplements, types of spread normally used on bread and other details of foods commonly eaten, to aid coding.

The completed diaries were coded by the same nutrition fieldworker using the computer program DIDO (Diet In, Data Out) originally developed by MRC Human Nutrition Research Unit and shown to improve speed and accuracy of dietary coding ${ }^{(20)}$. This program is designed for direct entry of dietary data and generates a food code and an associated weight for each item of food and drink recorded, and was extended to cover all foods eaten by the subjects. An advantage of this program is that new food codes can be added and portion sizes adjusted depending on the age group being assessed. When information on portion size was missing from the diary, average portion sizes for similar aged children were obtained from an analysis of weighed dietary intake from the National Diet and Nutrition Survey (NDNS) for 4- to 18-year-olds ${ }^{(21,22)}$. These portions were the same for both sexes.

The databank used for nutrient analysis included the fifth edition of McCance and Widdowson's food tables ${ }^{(23)}$ and supplements ${ }^{(24-31)}$. Additional up-to-date nutrient information was obtained from the NDNS database and manufacturers' information. The coded diaries were checked against the originals by a different nutrition fieldworker and any errors identified were corrected. Diaries that produced very high or low estimates for key nutrients were rechecked.

An in-house nutrient analysis program was used to generate the nutrients for each food the child ate. The average daily nutrient intakes and amount of various food groups were calculated. Nutrient intakes from vitamin supplements were not included in this analysis. Non-milk extrinsic sugars (NMES) were calculated based on the definition in the UK Dietary Reference Values (DRV) ${ }^{(32)}$. $\mathrm{Na}$ intake did not include salt added at the table and vegetables were coded as cooked without added salt; therefore it covered non-discretionary salt only.

\section{Maternal education and child antbropometry}

Highest maternal education level was derived from a questionnaire completed at 32 weeks of pregnancy, with answers grouped according to UK standards as follows: CSE or less (Certificate of Secondary Education; national school exams at age 16 years); vocational qualifications; O Levels (national school exams at 16 years, higher than CSE); A Levels (national school exams at 18 years); or degree. For the present analysis maternal education level was grouped as low (none, CSE or vocational), medium (O Levels) or high (A Level or degree). More up-to-date information about maternal education was not available. 
At the clinic, the child's height was measured to the last complete millimetre using a Harpenden stadiometer and his/her weight was measured to the nearest $50 \mathrm{~g}$ using a Tanita Body Fat Analyser (model TBF 305). BMI was calculated using the standard equation: $\mathrm{BMI}=$ weight $(\mathrm{kg}) /[\text { height }(\mathrm{m})]^{2}$. Overweight and obesity were defined using age- and sex-specific cut-off points identified by Cole et $a l^{(33)}$ using 1990 reference centiles, with underweight defined as in Cole et $a l^{(34)}$.

\section{Statistical metbods}

Nutrient intakes were checked visually for normality and those with skewed distributions were transformed to the natural logarithm prior to analysis to improve the normality of distributions. Energy adjustment was completed on all nutrients using the regression residual method recommended by Willett and Stampfer ${ }^{(35)}$. Weights of foods eaten per unit energy (MJ) were calculated. To compare intakes between maternal education groups, one-way ANOVA was conducted for nutrient intakes with linear regression performed to establish $P$ values for trend, and a Kruskal-Wallis test was used for food intakes because these data could not be normalised. All statistical analyses were performed using the SPSS for Windows statistical software package version 15 (SPSS Inc., Chicago, IL, USA).

\section{Misreporting}

Studies have shown that misreporting of energy intake (EI) can affect dietary surveys ${ }^{(36)}$; furthermore if EI is underestimated it is probable that intakes of other nutrients are also underestimated ${ }^{(37)}$. Misreporting of EI tends to be biased towards under-reporting and is more common in obese individuals ${ }^{(38)}$. It was important, therefore, to establish the degree to which misreporting occurred in the current sample. This was determined using an individualised method (which allows for moderate activity levels), by calculating the ratio of reported EI to estimated energy requirement $(\mathrm{EER})^{(39)}$. EER was calculated for each child based on his/her body weight, using separate equations for boys and girls, with an increment added for energy used in growth ${ }^{(40)}$. The validity of reported energy intake was assessed by comparing the calculated EER with EI: any EI less than $78 \cdot 45 \%$ or above $121.55 \%$ of EER was classified as under-reporting or over-reporting, respectively. A logistic regression analysis was conducted to establish if BMI status and maternal education had an independent effect on misreporting status.

\section{Energy and nutrient intakes - comparisons with national data and reference intakes}

The dietary intakes of the current sample were compared with similar data obtained from NDNS ${ }^{(21)}$. The latter survey of dietary habits and nutritional status studied a crosssectional sample of British children aged 4-18 years. Comparable data consisted of $7 \mathrm{~d}$ weighed food diaries collected in 1997 from 256 boys and 226 girls aged
7-10 years. Comparisons were made between the NDNS and the present study for the whole sample and the plausible reporters separately.

UK DRV ${ }^{(32)}$ were used to assess the adequacy of the recorded diets for most nutrients. At present, there is no official DRV for NSP (a measure of fibre intake); however, in adults the DRV is $18 \mathrm{~g}$ and intakes in children should be comparatively less due to smaller body sizes. NSP intakes have been calculated previously for 7-year-olds in ALSPAC $^{(41)}$, and the same method was used for the 10year-olds. $\mathrm{Na}$ is an essential nutrient but should not be taken in excess; the DRV is a Reference Nutrient Intake (RNI) set at $1200 \mathrm{mg} / \mathrm{d}^{(32)}$. Salt levels for children should be lower than for adults, with children aged 7-10 years consuming no more than $5 \mathrm{~g}(1966 \mathrm{mg} \mathrm{Na})$ daily ${ }^{(41,42)}$.

\section{Results}

\section{Response rates}

A total of 11868 children were eligible to attend the clinic for assessment at 10 years (mean age 10.6 years) and 7563 attended $(63.7 \%)$. Dietary diaries were available for 7474 (98.8\% of attendees); of these seventeen provided $4 \mathrm{~d}$ of intake $(24 \mathrm{~h}$ recall $+3 \mathrm{~d}), 5753$ provided $3 \mathrm{~d}, 694$ provided $2 \mathrm{~d}$ and 1010 provided $1 \mathrm{~d}$ only (usually a $24 \mathrm{~h}$ recall); all were included in the analysis. Table 1 illustrates characteristics of the children who did or did not attend the clinic and the anthropometric measures of attendees by sex. Girls were on average taller and heavier than boys. Of the mothers, $22 \%$ were classified as low educational status, $35 \%$ as medium and $43 \%$ as high. This showed a bias towards higher education compared with the non-attendees $(P<0 \cdot 001)$.

\section{Energy requirements and misreporting of energy intake}

Not all the sample could be classified by misreporting status owing to incomplete data, but classification was possible in $7433(98 \cdot 3 \%)$. The proportion of underreporters was similar in both sexes at about $36 \%$. Compared with the NDNS where the level of under-reporting increased substantially in the older age groups, from $27 \%$ in 7- to 10 -year-olds to $50 \%$ in 11 - to 14 -year-olds ${ }^{(21)}$, ALSPAC had an intermediate prevalence of underreporting but all of the children were at the maximum end of the 7-10-year age range. Over-reporters were identified, but numbers were small (3-4\% in ALSPAC $v .1-2 \%$ in NDNS). This meant that about $60 \%$ of the ALSPAC sample was considered to be plausibly reporting. Table 2 compares the weight status and maternal education level of the misreporting groups. The under-reporters were much more likely than the other two groups to be overweight or obese. Misreporting occurred more often in the lowest education group and least in the highest group. Logistic regression analysis showed that misreporting was independently associated with child BMI status but not 
Table 1 Characteristics of children who attended the research clinic at 10 years, Avon Longitudinal Study of Parents and Children

\begin{tabular}{|c|c|c|c|c|c|c|}
\hline & \multicolumn{4}{|c|}{ Attended clinic } & & \\
\hline & \multicolumn{2}{|c|}{ Boys ( $n$ 3735) } & \multicolumn{2}{|c|}{ Girls (n 3828) } & \multicolumn{2}{|c|}{ Did not attend clinic } \\
\hline & Mean or $n$ & SD or $\%$ & Mean or $n$ & SD or $\%$ & $n$ & $\%$ \\
\hline Diet diaries obtained & 3703 & & 3771 & & & \\
\hline Age (years) & $10 \cdot 6$ & 0.3 & $10 \cdot 6$ & 0.3 & & \\
\hline Height $(\mathrm{cm})$ & $143 \cdot 9$ & $6 \cdot 4$ & $144 \cdot 2$ & $7 \cdot 0$ & & \\
\hline Weight (kg) & $37 \cdot 6$ & $8 \cdot 2$ & $38 \cdot 6$ & $8 \cdot 9$ & & \\
\hline \multicolumn{7}{|c|}{ Maternal education level } \\
\hline Low & 752 & $20 \cdot 3$ & 748 & $19 \cdot 8$ & 2226 & $39 \cdot 4$ \\
\hline Medium & 1217 & $32 \cdot 9$ & 1207 & $32 \cdot 0$ & 1881 & $33 \cdot 3$ \\
\hline High & 1427 & $38 \cdot 5$ & 1477 & $39 \cdot 2$ & 1493 & $27 \cdot 4$ \\
\hline Missing & 307 & $8 \cdot 3$ & 339 & $9 \cdot 0$ & 56 & $1 \cdot 0$ \\
\hline
\end{tabular}

Data are expressed as mean and standard deviation for continuous variables or $n$ and \% for categorical variables.

Table 2 Reporting of dietary energy intake* by weight status, maternal education level and sex: 10-year-old children, Avon Longitudinal Study of Parents and Children

\begin{tabular}{lcccc}
\hline & Under-reporting (\%) & Plausible reporting (\%) & Over-reporting (\%) & $P$ value (logistic regression) \\
\hline BMI & & & & $<0 \cdot 001 /<0 \cdot 001 \dagger$ \\
Missing $(n$ 44) & $29 \cdot 5$ & $59 \cdot 1$ & $11 \cdot 4$ & \\
Underweight $(n$ 75) & $8 \cdot 0$ & $76 \cdot 0$ & $16 \cdot 0$ & \\
Healthy weight $(n$ 5707) & $27 \cdot 9$ & $67 \cdot 8$ & $0 \cdot 4$ & $0 \cdot 823 / 0 \cdot 053 \ddagger$ \\
Overweight $(n$ 1263) & $64 \cdot 1$ & $35 \cdot 7$ & - & \\
Obese $(n$ 344) & $82 \cdot 3$ & $17 \cdot 7$ & $4 \cdot 7$ & \\
Maternal education level & $42 \cdot 7$ & $52 \cdot 6$ & $4 \cdot 7$ & \\
Missing $(n$ 642) & $39 \cdot 7$ & $55 \cdot 6$ & $3 \cdot 3$ & \\
Low $(n$ 1497) & $35 \cdot 6$ & $61 \cdot 1$ & $2 \cdot 9$ & \\
Medium $(n$ 2410) & $34 \cdot 0$ & $63 \cdot 1$ & $4 \cdot 1$ & \\
High $(n$ 2884) & $36 \cdot 4$ & $59 \cdot 5$ & $3 \cdot 1$ & \\
Sex & $36 \cdot 4$ & $60 \cdot 5$ & & \\
Boys $(n$ 3685) & & & & \\
Girls $(n$ 3748) & & & & \\
\hline
\end{tabular}

${ }^{*}$ Overall prevalence of under-reporting, plausible reporting and over-reporting in the sample was $36 \cdot 2 \%, 59 \cdot 7 \%$ and $3 \cdot 6 \%$, respectively. $+P$ value in boys/girls for the difference in under-reporting prevalence by BMI group adjusting for the effect of maternal education level.

$\ddagger P$ value in boys/girls for the difference in under-reporting prevalence by maternal education group adjusting for the effect of BMI.

Table 3 Weight status by maternal education level: 10-year-old children, Avon Longitudinal Study of Parents and Children

\begin{tabular}{|c|c|c|c|c|c|}
\hline & \multicolumn{4}{|c|}{ Maternal education level } & \multirow[b]{2}{*}{$P$ value ( $\chi^{2}$ test) } \\
\hline & Low (\%) & Medium (\%) & High (\%) & Missing (\%) & \\
\hline BMI & & & & & $<0.001$ \\
\hline Underweight ( $n$ 48) & $21 \cdot 2$ & $31 \cdot 5$ & $38 \cdot 9$ & $8 \cdot 9$ & \\
\hline Healthy weight ( $n$ 2854) & $19 \cdot 0$ & $32 \cdot 5$ & $40 \cdot 7$ & $7 \cdot 8$ & \\
\hline Overweight ( $n$ 632) & $22 \cdot 4$ & $32 \cdot 2$ & $33 \cdot 9$ & $11 \cdot 6$ & \\
\hline Obese $(n$ 173) & $30 \cdot 3$ & $31 \cdot 1$ & $27 \cdot 5$ & $11 \cdot 3$ & \\
\hline
\end{tabular}

maternal education. Table 3 shows the weight status of the children by maternal education group; an excess of children in the low education group were classified as obese. The prevalence of underweight was similar across all maternal education groups, but more underweight girls had mothers of high education.

\section{Food and nutrients according to misreporting status}

By definition there were major differences in mean energy and nutrient intakes between the misreporting status groups (data not shown). Table 4 shows the overall mean food intakes in the under-reporters compared with plausible reporters; there were too few over-reporters to allow a separate comparison. There were differences in recorded intakes in both sexes of biscuits, cakes, puddings, rice and pasta, whole milk, chocolate, sweets and sweet spreads such that the under-reporters recorded substantially lower intakes (all $P<0 \cdot 001$ ). Intakes of meat and meat products and fish were not different between the groups. No differences were seen between under-reporters and plausible reporters for vegetables in either sex, but there were differences for cooked vegetables in girls $(P=0001)$ and for fruit and fruit juice for boys only $(P<0 \cdot 001)$. 
Table 4 Overall mean food intakes and percentage of consumers by under-reporting and plausibly reporting and sex, and significance of the difference comparing under-reporters and plausible reporters in boys and girls separately using the Kruskal-Wallis test: 10-year-old children, Avon Longitudinal Study of Parents and Children

\begin{tabular}{|c|c|c|c|c|c|c|c|c|c|c|}
\hline \multirow[b]{3}{*}{ Food Item } & \multicolumn{4}{|c|}{ Under-reporters } & \multicolumn{4}{|c|}{ Plausible reporters } & & \\
\hline & \multicolumn{2}{|c|}{ Boys ( $n$ 1341) } & \multicolumn{2}{|c|}{ Girls (n 1365) } & \multicolumn{2}{|c|}{ Boys ( $n$ 2194) } & \multicolumn{2}{|c|}{ Girls (n 2268) } & \multicolumn{2}{|c|}{$\begin{array}{c}P \text { value (under-reporters } \\
V . \text { plausible reporters) }\end{array}$} \\
\hline & $\begin{array}{l}\text { Intake } \\
(\mathrm{g})\end{array}$ & $\begin{array}{c}\% \text { of } \\
\text { consumers }\end{array}$ & $\begin{array}{l}\text { Intake } \\
(\mathrm{g})\end{array}$ & $\begin{array}{c}\% \text { of } \\
\text { consumers }\end{array}$ & $\begin{array}{l}\text { Intake } \\
(\mathrm{g})\end{array}$ & $\begin{array}{c}\% \text { of } \\
\text { consumers }\end{array}$ & $\begin{array}{l}\text { Intake } \\
(\mathrm{g})\end{array}$ & $\begin{array}{c}\% \text { of } \\
\text { consumers }\end{array}$ & $\begin{array}{l}\text { Boys } \\
(n 3535)\end{array}$ & $\begin{array}{c}\text { Girls } \\
(n 3633)\end{array}$ \\
\hline \multicolumn{11}{|l|}{ Cereal products } \\
\hline High-fibre breakfast cereals & $15 \cdot 2$ & 47 & $10 \cdot 1$ & 39 & $17 \cdot 7$ & 50 & $13 \cdot 6$ & 48 & $0 \cdot 731$ & $<0.001$ \\
\hline Other breakfast cereals & $12 \cdot 7$ & 49 & $9 \cdot 1$ & 43 & $15 \cdot 4$ & 53 & $11 \cdot 5$ & 50 & 0.951 & 0.038 \\
\hline White bread & $57 \cdot 4$ & 82 & $50 \cdot 9$ & 84 & $67 \cdot 3$ & 87 & $62 \cdot 0$ & 89 & 0.005 & 0.068 \\
\hline Brown \& wholemeal bread & $11 \cdot 0$ & 22 & $8 \cdot 8$ & 21 & $13 \cdot 1$ & 25 & $10 \cdot 6$ & 24 & $0 \cdot 321$ & 0.064 \\
\hline Other bread & 3.9 & 12 & 4.9 & 16 & $5 \cdot 8$ & 17 & $5 \cdot 9$ & 18 & $<0.001$ & $0 \cdot 175$ \\
\hline Biscuits & $16 \cdot 1$ & 68 & $13 \cdot 1$ & 68 & $23 \cdot 1$ & 79 & $20 \cdot 2$ & 80 & $<0.001$ & $<0.001$ \\
\hline Buns, cakes \& pastries & $19 \cdot 3$ & 53 & $18 \cdot 5$ & 56 & $31 \cdot 0$ & 69 & $31 \cdot 1$ & 71 & $<0.001$ & $<0.001$ \\
\hline Puddings & $36 \cdot 5$ & 59 & $31 \cdot 4$ & 54 & $50 \cdot 0$ & 68 & $46 \cdot 8$ & 69 & $<0.001$ & $<0.001$ \\
\hline Rice \& pasta & $75 \cdot 7$ & 75 & $72 \cdot 8$ & 79 & $80 \cdot 1$ & 78 & $77 \cdot 1$ & 79 & $<0.001$ & $<0.001$ \\
\hline \multicolumn{11}{|l|}{ Meat \& meat products } \\
\hline Meat & $42 \cdot 0$ & 72 & $38 \cdot 2$ & 71 & $46 \cdot 6$ & 75 & $42 \cdot 3$ & 72 & 0.089 & 0.023 \\
\hline Meat pies \& pasties & $6 \cdot 8$ & 15 & $5 \cdot 8$ & 15 & $8 \cdot 8$ & 18 & $7 \cdot 7$ & 17 & 0.022 & $0 \cdot 344$ \\
\hline Sausages, burgers \& kebabs & $15 \cdot 4$ & 45 & $12 \cdot 5$ & 41 & $18 \cdot 1$ & 49 & $13 \cdot 3$ & 42 & $0 \cdot 656$ & $0 \cdot 131$ \\
\hline Breaded chicken or turkey & $9 \cdot 8$ & 26 & $9 \cdot 4$ & 27 & $10 \cdot 3$ & 26 & $10 \cdot 5$ & 28 & 0.753 & 0.553 \\
\hline Chicken \& turkey dishes & $23 \cdot 1$ & 51 & $20 \cdot 1$ & 48 & $26 \cdot 0$ & 54 & $24 \cdot 0$ & 53 & $0 \cdot 777$ & 0.549 \\
\hline Other meat \& meat dishes & $3 \cdot 0$ & 16 & $2 \cdot 7$ & 14 & $3 \cdot 6$ & 19 & $2 \cdot 9$ & 16 & 0.028 & $0 \cdot 149$ \\
\hline \multicolumn{11}{|l|}{ Fish } \\
\hline Coated white fish & $7 \cdot 1$ & 18 & $6 \cdot 2$ & 18 & $8 \cdot 1$ & 20 & $6 \cdot 4$ & 18 & 0.346 & 0.259 \\
\hline Other fish & $2 \cdot 3$ & 7 & $1 \cdot 5$ & 5 & $1 \cdot 8$ & 5 & $2 \cdot 0$ & 6 & 0.041 & 0.265 \\
\hline Oily fish & $4 \cdot 2$ & 14 & $5 \cdot 2$ & 18 & $4 \cdot 3$ & 14 & $5 \cdot 2$ & 19 & 0.996 & 0.740 \\
\hline \multicolumn{11}{|l|}{ Vegetables } \\
\hline Baked beans & $21 \cdot 2$ & 33 & $16 \cdot 0$ & 30 & $24 \cdot 2$ & 37 & $19 \cdot 0$ & 32 & $0 \cdot 129$ & 0.622 \\
\hline Raw vegetables & $13 \cdot 3$ & 35 & $18 \cdot 2$ & 46 & $15 \cdot 5$ & 41 & $20 \cdot 8$ & 52 & 0.041 & $0 \cdot 177$ \\
\hline Cooked vegetables & $42 \cdot 4$ & 71 & $42 \cdot 9$ & 73 & $51 \cdot 8$ & 79 & $51 \cdot 4$ & 80 & $0 \cdot 169$ & 0.001 \\
\hline Legumes & $1 \cdot 2$ & 4 & 0.9 & 4 & 0.9 & 4 & $1 \cdot 1$ & 4 & 0.972 & $0 \cdot 351$ \\
\hline \multicolumn{11}{|l|}{ Fruit } \\
\hline Fresh fruit & $51 \cdot 4$ & 58 & $60 \cdot 9$ & 68 & $66 \cdot 4$ & 70 & $73 \cdot 5$ & 77 & $<0.001$ & 0.455 \\
\hline Canned fruit & $1 \cdot 9$ & 6 & $2 \cdot 2$ & 9 & $3 \cdot 0$ & 7 & $3 \cdot 7$ & 10 & 0.032 & 0.002 \\
\hline Fruit juice & 94.9 & 47 & $95 \cdot 4$ & 52 & $131 \cdot 9$ & 57 & $123 \cdot 6$ & 60 & $<0.001$ & 0.009 \\
\hline Nuts & $1 \cdot 4$ & 11 & $1 \cdot 0$ & 11 & $1 \cdot 8$ & 14 & $1 \cdot 6$ & 16 & 0.047 & $<0.001$ \\
\hline \multicolumn{11}{|l|}{ Potatoes } \\
\hline Fried/roast potatoes or chips & $58 \cdot 1$ & 74 & $55 \cdot 2$ & 76 & $66 \cdot 9$ & 81 & $59 \cdot 2$ & 77 & 0.085 & $<0.001$ \\
\hline Other potatoes & $30 \cdot 7$ & 44 & $29 \cdot \overline{6}$ & 49 & $35 \cdot 0$ & 50 & $34 \cdot 8$ & 53 & $0 \cdot 267$ & 0.813 \\
\hline \multicolumn{11}{|l|}{ Dairy products } \\
\hline Whole milk & $49 \cdot 5$ & 26 & $32 \cdot 1$ & 23 & $96 \cdot 6$ & 37 & $64 \cdot 6$ & 32 & $<0.001$ & $<0.001$ \\
\hline Semi-skimmed milk & $130 \cdot 0$ & 63 & $93 \cdot 6$ & 60 & $156 \cdot 3$ & 61 & $133 \cdot 5$ & 62 & 0.038 & 0.022 \\
\hline Skimmed milk & $8 \cdot 1$ & 7 & $6 \cdot 9$ & 5 & $5 \cdot 3$ & 4 & $6 \cdot 4$ & 5 & $<0.001$ & $0 \cdot 648$ \\
\hline Soya milk & $0 \cdot 4$ & $0 \cdot 4$ & $0 \cdot 8$ & 0.4 & $1 \cdot 3$ & 1 & $0 \cdot 8$ & 1 & $0 \cdot 140$ & $0 \cdot 713$ \\
\hline Goat's/sheep's milk & $1 \cdot 0$ & 0.4 & 0.2 & $0 \cdot 1$ & 0.5 & 0.2 & 0.5 & 0.2 & 0.431 & $0 \cdot 622$ \\
\hline Other milk/cream & $1 \cdot 2$ & 11 & 0.9 & 12 & 1.9 & 16 & 1.9 & 17 & 0.001 & $<0.001$ \\
\hline Yoghurt/fromage frais & $29 \cdot 3$ & 39 & $27 \cdot 1$ & 39 & $35 \cdot 2$ & 45 & $34 \cdot 0$ & 47 & $0 \cdot 127$ & $0 \cdot 011$ \\
\hline Eggs & $8 \cdot 8$ & 25 & $8 \cdot 2$ & 29 & $9 \cdot 6$ & 28 & $9 \cdot 0$ & 30 & 0.491 & $0 \cdot 711$ \\
\hline Cheese & $10 \cdot 0$ & 47 & $9 \cdot 8$ & 51 & $13 \cdot 3$ & 54 & $13 \cdot 6$ & 60 & 0.006 & 0.005 \\
\hline
\end{tabular}




\section{Nutrient intakes according to maternal education level}

Table 5 examines energy-adjusted mean nutrient intakes according to maternal educational level in plausible reporters only. There were no clear differences between education groups for macronutrients, except in sugars where total sugars showed a gradient. There was no association between maternal education and NMES, but those from high education groups consumed more intrinsic sugars (contained within fruit and vegetables). We observed marked differences in micronutrient intakes, especially for vitamin $\mathrm{C}$, carotene and retinol equivalents, such that as maternal education fell the amount of nutrient decreased by $23 \%, 15 \%$ and $12 \%$ respectively. Folate, $\mathrm{Mg}$ and riboflavin were $7 \%$ higher in the high $v$. low education group and $\mathrm{Ca}, \mathrm{Fe}$ and vitamin $\mathrm{B}_{12}$ showed a similar small gradient; Na levels were highest in the low education group and fell with education level (all $P<0 \cdot 001)$.

\section{Food group intakes according to maternal education level}

Table 6 illustrates energy-adjusted mean intakes of food items for the plausible reporters only according to maternal education level. All types of fruit and vegetables (except baked beans) had a small gradient towards the highest education group, particularly fresh fruit $(P<0 \cdot 001)$ and fruit juice $(P<0 \cdot 001)$. Consumption was fairly similar for meat and poultry; however, meat products such as meat pies $(P<0 \cdot 001)$ and sausages, burgers and kebabs $(P<0 \cdot 001)$ showed a positive trend towards the lower education group. Intakes of oily fish in particular showed an educational gradient $(P<0 \cdot 001)$. White bread fell with education level $(P=0 \cdot 046)$, as brown and wholemeal bread rose $(P<0 \cdot 001)$, however over $80 \%$ of children consumed white bread. A similar pattern was observed for milk; intake of whole milk fell with education level $(P=0 \cdot 001)$, while that of semi-skimmed milk increased $(P<0 \cdot 001)$. Sugar confectionery decreased from low to high education groups $(P=0 \cdot 006)$, and chocolate confectionery was highest in the middle education group $(P<0 \cdot 001)$. Intakes of sugar confectionery, chocolate confectionery and savoury snacks were high in all education groups. Consumption of biscuits fell with maternal education $(P=0 \cdot 002)$; however, that of buns, cakes and pastries rose $(P<0 \cdot 001)$. Diet soft drinks were consumed in greater amounts than sugar-sweetened ones and showed a positive trend towards the lowest education group $(P<0 \cdot 001)$.

\section{Nutrient intakes and comparisons with national data}

Comparisons of energy and nutrient intakes by sex for the whole ALSPAC sample with NDNS children aged 7-10 years and for plausible reporters in ALSPAC separately (Supplementary Table 1) showed that intakes of 
Table 5 Energy and energy-adjusted nutrient mean intakes, and their $95 \%$ confidence intervals, for plausible reporters only by maternal education level, using ANOVA to compare diets and linear regression to obtain $P$ for trend: 10 -year-old children, Avon Longitudinal Study of Parents and Children

\begin{tabular}{|c|c|c|c|c|c|c|c|}
\hline \multirow[b]{3}{*}{ Nutrient } & \multicolumn{6}{|c|}{ Maternal education level } & \multirow[b]{3}{*}{$P$ for trend } \\
\hline & \multicolumn{2}{|c|}{ Low (n 832) } & \multicolumn{2}{|c|}{ Medium (n 1472) } & \multicolumn{2}{|c|}{ High (n 1820) } & \\
\hline & Mean & $95 \% \mathrm{Cl}$ & Mean & $95 \% \mathrm{Cl}$ & Mean & $95 \% \mathrm{Cl}$ & \\
\hline Energy (MJ) & $8 \cdot 39$ & $8 \cdot 31,8 \cdot 48$ & $8 \cdot 40$ & $8 \cdot 34,8 \cdot 46$ & $8 \cdot 38$ & $8 \cdot 32,8 \cdot 43$ & 0.716 \\
\hline Protein $(\mathrm{g})$ & $59 \cdot 8$ & $59 \cdot 0,60 \cdot 6$ & $60 \cdot 1$ & $59 \cdot 6,60 \cdot 6$ & $61 \cdot 2 \ddagger$ & $60 \cdot 7,61 \cdot 7$ & $<0.001$ \\
\hline Fat $(\mathrm{q})$ & $75 \cdot 7 \ddagger$ & $75 \cdot 0,76 \cdot 4$ & $74 \cdot 5$ & $74 \cdot 0,75 \cdot 0$ & $73 \cdot 4$ & $72 \cdot 9,73 \cdot 8$ & $<0.001$ \\
\hline Saturated fat $(\mathrm{g})$ & $29 \cdot 1 \ddagger$ & $28 \cdot 6,29 \cdot 5$ & $28 \cdot 5$ & $28 \cdot 2,28 \cdot 8$ & $28 \cdot 3$ & $28 \cdot 0,28 \cdot 6$ & 0.003 \\
\hline Monounsaturated fat $(\mathrm{g})$ & $25 \cdot 7 \ddagger$ & $25 \cdot 4,26 \cdot 0$ & $25 \cdot 1$ & $24 \cdot 9,25 \cdot 3$ & $24 \cdot 4$ & $24 \cdot 2,24 \cdot 6$ & $<0.001$ \\
\hline Polyunsaturated fat (g) & $12 \cdot 2 \ddagger$ & $12 \cdot 0,12 \cdot 5$ & $12 \cdot 1$ & $11 \cdot 9,12 \cdot 3$ & $11 \cdot 8$ & $11 \cdot 6,12 \cdot 0$ & 0.002 \\
\hline Carbohydrate $(\mathrm{g})$ & $243^{2+}$ & 241,244 & 246 & 245,247 & $247 \ddagger$ & 246,248 & $<0.001$ \\
\hline Total sugars $(\mathrm{g})$ & 107 & 105,109 & 111 & 110,113 & $114 \ddagger$ & 112,115 & $<0.001$ \\
\hline NMES $(\mathrm{g})$ & 83 & 81,85 & 85 & 84,84 & 85 & 84,86 & $0 \cdot 103$ \\
\hline Starch $(\mathrm{g})$ & 131 & 130,133 & 129 & 128,131 & 129 & 128,130 & 0.014 \\
\hline NSP $(g)$ & $10 \cdot 8$ & $10 \cdot 6,11 \cdot 0$ & $11 \cdot 0$ & $10 \cdot 8,11 \cdot 1$ & $11 \cdot 5 \ddagger$ & $11 \cdot 5,11 \cdot 8$ & $<0.001$ \\
\hline Carotene $(\mu \mathrm{g})$ & 1616 & 1520,1716 & 1722 & 1650,1795 & $1863 \ddagger$ & 1795,1931 & $<0.001$ \\
\hline Retinol $(\mu \mathrm{g})$ & 280 & 269, 292 & 289 & 282,297 & $304 \ddagger$ & 297,311 & $<0.001$ \\
\hline Retinol equivalents $(\mu \mathrm{g})^{\star} \dagger$ & 563 & 542,585 & 588 & 574,603 & $628 \ddagger$ & 615,642 & $<0.001$ \\
\hline Thiamin (mg)† & $1 \cdot 33$ & $1 \cdot 30,1 \cdot 36$ & $1 \cdot 37$ & $1 \cdot 35,1 \cdot 40$ & $1 \cdot 43 \ddagger$ & $1 \cdot 40,1 \cdot 45$ & $<0.001$ \\
\hline Riboflavin (mg) & $1 \cdot 41$ & $1 \cdot 38,1 \cdot 45$ & $1 \cdot 44$ & $1 \cdot 42,1 \cdot 47$ & $1.52 \ddagger$ & $1.49,1.54$ & $<0.001$ \\
\hline Niacin equivalents $(\mathrm{mg})^{*}$ & $28 \cdot 0$ & $27 \cdot 6,28 \cdot 5$ & $28 \cdot 5$ & $28 \cdot 2,28 \cdot 8$ & $29 \cdot 3 \ddagger$ & $29 \cdot 0,29 \cdot 5$ & $<0.001$ \\
\hline Vitamin $B_{6}(\mathrm{mg})$ & $1 \cdot 79$ & $1 \cdot 76,1 \cdot 83$ & $1 \cdot 82$ & $1 \cdot 80,1 \cdot 85$ & $1 \cdot 84 \ddagger$ & $1 \cdot 82,1 \cdot 86$ & 0.028 \\
\hline Vitamin $B_{12}(\mathrm{mg}) \dagger$ & $3 \cdot 11$ & $3 \cdot 01,3 \cdot 21$ & $3 \cdot 10$ & $3 \cdot 03,3 \cdot 18$ & $3 \cdot 31 \ddagger$ & $3 \cdot 25,3 \cdot 39$ & $<0.001$ \\
\hline Folate $(\mu \mathrm{g})$ & 195 & 191,199 & 200 & 197,203 & $211 \ddagger$ & 208,214 & $<0.001$ \\
\hline Vitamin C (mg)t & $67 \cdot 3$ & $64 \cdot 0,70 \cdot 8$ & $74 \cdot 0$ & $71 \cdot 3,76 \cdot 7$ & $88 \cdot 0 \ddagger$ & $85 \cdot 3,90 \cdot 6$ & $<0.001$ \\
\hline Vitamin $D(\mu \mathrm{g}) \dagger$ & $2 \cdot 35$ & $2 \cdot 27,2 \cdot 42$ & $2 \cdot 43$ & $2 \cdot 37,2 \cdot 48$ & $2 \cdot 50 \ddagger$ & $2 \cdot 45,2 \cdot 56$ & 0.001 \\
\hline $\mathrm{Na}(\mathrm{mg})$ & $2559 \ddagger$ & 2523,2596 & 2547 & 2521,2574 & 2486 & 2463,2508 & $<0.001$ \\
\hline $\mathrm{Ca}(\mathrm{mg})$ & 721 & 706,736 & 739 & 728,750 & $759 \ddagger$ & 749,769 & $<0.001$ \\
\hline $\mathrm{Mg}(\mathrm{mg})$ & 201 & 198,203 & 204 & 203,206 & $214 \ddagger$ & 212,215 & $<0.001$ \\
\hline $\mathrm{K}(\mathrm{mg})$ & 2356 & 2325,2387 & 2357 & 2334,2381 & $2393 \ddagger$ & 2372,2414 & 0.024 \\
\hline $\mathrm{Fe}(\mathrm{mg})$ & 8.5 & $8 \cdot 3,8 \cdot 6$ & 8.5 & $8 \cdot 4,8 \cdot 6$ & $8 \cdot 9 \ddagger$ & $8 \cdot 9,9 \cdot 0$ & $<0.001$ \\
\hline $\mathrm{Zn}(\mathrm{mg})$ & $6 \cdot 46$ & $6 \cdot 35,6 \cdot 58$ & $6 \cdot 42$ & $6 \cdot 34,6 \cdot 50$ & $6 \cdot 69 \ddagger$ & $6 \cdot 61,6 \cdot 76$ & $<0.001$ \\
\hline $\mathrm{Cu}(\mathrm{mg})$ & 0.78 & $0.77,0.79$ & 0.80 & $0.79,0.81$ & $0.83 \ddagger$ & $0.82,0.84$ & $<0.001$ \\
\hline Se $(\mu g)$ & $54 \cdot 3$ & $53 \cdot 1,55 \cdot 5$ & $54 \cdot 5$ & $53 \cdot 7,55 \cdot 4$ & $56 \cdot 9 \ddagger$ & $56 \cdot 2,57 \cdot 7$ & $<0.001$ \\
\hline lodine $(\mu \mathrm{g}) \dagger$ & 111 & 108,113 & 114 & 112,116 & $116 \ddagger$ & 115,118 & $<0.001$ \\
\hline
\end{tabular}

NMES, non-milk extrinsic sugars.

${ }^{*}$ Retinol equivalents $=$ carotene $/ 6+$ retinol; niacin equivalents $=$ niacin + tryptophan $/ 60$. †Transformed to the natural logarithm, geometric mean and confidence intervals. ‡Direction of trend for maternal education groups $(P<0 \cdot 01)$.

energy and most nutrients were slightly higher in ALSPAC than in NDNS for both sexes, but this was likely to be explained by the higher average age in the ALSPAC sample. Exceptions to this were observed for vitamin $\mathrm{B}_{12}$, vitamin $\mathrm{C}$, vitamin $\mathrm{D}, \mathrm{Fe}$ and iodine in both sexes; also vitamin $\mathrm{B}_{6}$ in girls. Energy intake and intakes of most nutrients were higher in boys than girls in both studies. Compared with DRV there was no evidence of inadequacy for any nutrient (mean intakes of all vitamins and minerals exceeded the RNI), except NSP; however, intakes of saturated fat, NMES and $\mathrm{Na}$ (non-discretionary) were substantially higher than recommended.

\section{Discussion}

In this large prospective study of diet in 10-year-old children we found a social gradient in diet quality, such that as maternal education increased diet quality improved. This was particularly true of the types of foods eaten.
Girls were taller and heavier than the boys which is typical at this age ${ }^{(1)}$; however, boys had higher intakes of energy and macronutrients and similar levels of underreporting. It is likely that the higher energy intake in boys was due to higher activity levels since objective physical activity data, obtained from ALSPAC at age 11 years, have shown that boys were more active than girls and spent more time in moderate-to-vigorous physical activity ${ }^{(43)}$. In the present sample, more children with mothers in the lowest education group were classified as obese, while those classified as underweight or healthy weight were more likely to have mothers in the highest education group.

We used the method of Torun to estimate levels of misreporting of dietary intakes; this allows sex and body weight to be considered but includes only a standard increment for moderate physical activity. Levels of underreporting were high (36\%) and over-reporting low (4\%); therefore to assess differences in foods recorded we looked at under-reporters compared with plausible reporters. Under-reporters consistently recorded substantially less 
Table 6 Energy-adjusted overall mean intakes and their standard error, and percentage of consumers of food items, for plausible reporters only by maternal education level, using the Kruskal-Wallis test to compare diets: 10-year-old children, Avon Longitudinal Study of Parents and Children

\begin{tabular}{|c|c|c|c|c|c|c|c|c|c|c|}
\hline \multirow[b]{4}{*}{ Food item } & \multicolumn{9}{|c|}{ Maternal education level } & \multirow{4}{*}{$\begin{array}{c}P \text { value } \\
\text { (Kruskal-Wallis } \\
\text { test) }\end{array}$} \\
\hline & \multicolumn{3}{|c|}{ Low ( $n$ 832) } & \multicolumn{3}{|c|}{ Medium (n 1472) } & \multicolumn{3}{|c|}{ High (n 1820) } & \\
\hline & \multicolumn{2}{|c|}{ Intake (g/MJ) } & \multirow{2}{*}{$\begin{array}{c}\% \text { of } \\
\text { consumers }\end{array}$} & \multicolumn{2}{|c|}{ Intake (g/MJ) } & \multirow{2}{*}{$\begin{array}{c}\% \text { of } \\
\text { consumers }\end{array}$} & \multicolumn{2}{|c|}{ Intake (g/MJ) } & \multirow{2}{*}{$\begin{array}{c}\% \text { of } \\
\text { consumers }\end{array}$} & \\
\hline & Mean & SE & & Mean & SE & & Mean & SE & & \\
\hline \multicolumn{11}{|l|}{ Cereal products } \\
\hline High-fibre breakfast cereals & $1 \cdot 79$ & 0.11 & $45 \cdot 3$ & $1 \cdot 69$ & 0.07 & $47 \cdot 6$ & $2 \cdot 14^{*}$ & 0.08 & $49 \cdot 3$ & $<0.001$ \\
\hline Other breakfast cereals & 1.59 & 0.07 & $50 \cdot 0$ & $1 \cdot 65$ & 0.06 & $52 \cdot 5$ & $1 \cdot 56$ & 0.05 & $50 \cdot 4$ & 0.493 \\
\hline White bread & $7 \cdot 88$ & 0.20 & $86 \cdot 8$ & $7 \cdot 90$ & $0 \cdot 14$ & $88 \cdot 8$ & $7 \cdot 42$ & $0 \cdot 12$ & $87 \cdot 9$ & 0.046 \\
\hline Brown \& wholemeal bread & 1.09 & 0.09 & $18 \cdot 5$ & $1 \cdot 21$ & 0.08 & $21 \cdot 1$ & $1 \cdot 72^{*}$ & 0.08 & $30 \cdot 1$ & $<0.001$ \\
\hline Other bread & 0.45 & 0.05 & $13 \cdot 1$ & 0.63 & 0.05 & $15 \cdot 9$ & $0 \cdot 87^{*}$ & 0.05 & $21 \cdot 4$ & $<0.001$ \\
\hline Biscuits & $2 \cdot 79^{\star}$ & 0.09 & $78 \cdot 8$ & $2 \cdot 74$ & 0.07 & $79 \cdot 9$ & $2 \cdot 41$ & 0.05 & $79 \cdot 6$ & 0.002 \\
\hline Buns, cakes \& pastries & $3 \cdot 10$ & $0 \cdot 12$ & $62 \cdot 3$ & 3.51 & $0 \cdot 10$ & $68 \cdot 5$ & $4 \cdot 09^{*}$ & 0.09 & $74 \cdot 3$ & $<0.001$ \\
\hline Puddings & $5 \cdot 69$ & 0.22 & $65 \cdot 6$ & $5 \cdot 85$ & $0 \cdot 16$ & $69 \cdot 2$ & $5 \cdot 70$ & $0 \cdot 14$ & $69 \cdot 8$ & 0.677 \\
\hline Rice \& pasta & $8 \cdot 87$ & 0.32 & $76 \cdot 1$ & $8 \cdot 84$ & 0.23 & $76 \cdot 6$ & $10 \cdot 13^{\star}$ & 0.22 & $81 \cdot 4$ & $<0.001$ \\
\hline \multicolumn{11}{|l|}{ Meat \& meat products } \\
\hline Meat & $5 \cdot 40$ & 0.21 & $73 \cdot 1$ & $5 \cdot 23$ & $0 \cdot 15$ & $73 \cdot 2$ & $5 \cdot 36$ & $0 \cdot 14$ & $74 \cdot 1$ & 0.396 \\
\hline Meat pies \& pasties & $1 \cdot 28^{*}$ & $0 \cdot 11$ & $20 \cdot 9$ & $1 \cdot 06$ & 0.07 & $19 \cdot 5$ & $0 \cdot 76$ & 0.05 & $14 \cdot 7$ & $<0.001$ \\
\hline Sausages, burgers \& kebabs & $2 \cdot 25^{\star}$ & $0 \cdot 12$ & $49 \cdot 5$ & 1.92 & 0.08 & $46 \cdot 6$ & $1 \cdot 61$ & 0.06 & $41 \cdot 9$ & $<0.001$ \\
\hline Breaded chicken or turkey & $1 \cdot 30$ & $0 \cdot 10$ & $27 \cdot 3$ & $1 \cdot 44^{\star}$ & 0.07 & $30 \cdot 8$ & $1 \cdot 07$ & 0.06 & $24 \cdot 6$ & $<0.001$ \\
\hline Chicken \& turkey dishes & $3 \cdot 01$ & $0 \cdot 16$ & $51 \cdot 1$ & $3 \cdot 02$ & $0 \cdot 11$ & $54 \cdot 6$ & $2 \cdot 92$ & 0.09 & $53 \cdot 4$ & 0.478 \\
\hline Other meat and meat dishes & 0.43 & 0.05 & $16 \cdot 6$ & $0 \cdot 38$ & 0.03 & $16 \cdot 4$ & 0.37 & 0.03 & $18 \cdot 3$ & 0.960 \\
\hline \multicolumn{11}{|l|}{ Fish } \\
\hline Coated white fish & 0.91 & 0.08 & $20 \cdot 0$ & $0 \cdot 86$ & 0.06 & $19 \cdot 1$ & 0.87 & 0.05 & $18 \cdot 4$ & 0.677 \\
\hline Other fish & $0 \cdot 14$ & 0.03 & 3.5 & $0 \cdot 18$ & 0.03 & $4 \cdot 3$ & $0 \cdot 30^{*}$ & 0.04 & $7 \cdot 4$ & $<0.001$ \\
\hline Oily fish & $0 \cdot 39$ & 0.06 & $10 \cdot 6$ & 0.43 & 0.04 & $13 \cdot 7$ & $0 \cdot 76^{\star}$ & 0.04 & 22.3 & $<0.001$ \\
\hline \multicolumn{11}{|l|}{ Vegetables } \\
\hline Baked beans & $2 \cdot 98^{\star}$ & $0 \cdot 19$ & $39 \cdot 3$ & $2 \cdot 65$ & $0 \cdot 13$ & $34 \cdot 3$ & $2 \cdot 33$ & $0 \cdot 11$ & $32 \cdot 9$ & 0.004 \\
\hline Raw vegetables & $1 \cdot 55$ & $0 \cdot 11$ & $35 \cdot 9$ & $1 \cdot 85$ & 0.09 & $40 \cdot 8$ & $2 \cdot 84^{\star}$ & $0 \cdot 10$ & $56 \cdot 0$ & $<0.001$ \\
\hline Cooked vegetables & $5 \cdot 84$ & 0.22 & $73 \cdot 6$ & $6 \cdot 17$ & $0 \cdot 16$ & $78 \cdot 0$ & $6 \cdot 52^{*}$ & $0 \cdot 14$ & $82 \cdot 7$ & $<0.001$ \\
\hline Legumes & 0.07 & 0.03 & 1.9 & $0 \cdot 10$ & 0.02 & $3 \cdot 0$ & $0 \cdot 17^{\star}$ & 0.02 & $5 \cdot 5$ & $<0.001$ \\
\hline \multicolumn{11}{|l|}{ Fruit } \\
\hline Fresh fruit & $6 \cdot 05$ & 0.28 & $59 \cdot 9$ & $7 \cdot 82$ & 0.24 & $71 \cdot 1$ & $9 \cdot 94^{*}$ & $0 \cdot 21$ & $81 \cdot 7$ & $<0.001$ \\
\hline Canned fruit & $0 \cdot 30$ & 0.05 & $7 \cdot 0$ & 0.42 & 0.05 & $8 \cdot 4$ & 0.39 & 0.04 & $9 \cdot 1$ & $0 \cdot 190$ \\
\hline Fruit juice & $11 \cdot 58$ & 0.60 & $47 \cdot 1$ & $13 \cdot 70$ & 0.47 & $54 \cdot 9$ & $18 \cdot 52^{*}$ & 0.49 & $66 \cdot 8$ & $<0.001$ \\
\hline Nuts & $0 \cdot 12$ & 0.02 & $9 \cdot 5$ & $0 \cdot 15$ & 0.02 & $12 \cdot 2$ & $0 \cdot 28^{*}$ & 0.02 & $19 \cdot 8$ & $<0.001$ \\
\hline \multicolumn{11}{|l|}{ Potatoes } \\
\hline Fried/roast potatoes or chips & $9 \cdot 33^{*}$ & 0.24 & 84.9 & $8 \cdot 02$ & $0 \cdot 17$ & $82 \cdot 5$ & $6 \cdot 36$ & $0 \cdot 14$ & $73 \cdot 2$ & $<0.001$ \\
\hline Other potatoes & $4 \cdot 00$ & 0.20 & $47 \cdot 2$ & $4 \cdot 12$ & $0 \cdot 14$ & $51 \cdot 1$ & $4 \cdot 41$ & $0 \cdot 13$ & $53 \cdot 9$ & 0.024 \\
\hline \multicolumn{11}{|l|}{ Dairy products } \\
\hline Whole milk & $10 \cdot 37^{\star}$ & 0.63 & $37 \cdot 4$ & $9 \cdot 70$ & 0.46 & $35 \cdot 7$ & $8 \cdot 53$ & 0.39 & $30 \cdot 7$ & 0.001 \\
\hline Semi-skimmed milk & $15 \cdot 75$ & 0.73 & $57 \cdot 6$ & $16 \cdot 58$ & 0.53 & $60 \cdot 1$ & $18 \cdot 35^{\star}$ & $0 \cdot 50$ & $64 \cdot 8$ & $<0.001$ \\
\hline Skimmed milk & $0 \cdot 72$ & $0 \cdot 16$ & $4 \cdot 2$ & $0 \cdot 78$ & $0 \cdot 12$ & $4 \cdot 7$ & $0 \cdot 62$ & 0.09 & $4 \cdot 7$ & $0 \cdot 848$ \\
\hline Soya milk & $0 \cdot 11$ & 0.06 & $0 \cdot 4$ & 0.04 & 0.02 & $0 \cdot 3$ & $0 \cdot 18$ & 0.05 & $0 \cdot 1$ & 0.023 \\
\hline Goat's/sheep's milk & 0.08 & 0.08 & $0 \cdot 1$ & 0.02 & 0.02 & $0 \cdot 1$ & 0.09 & 0.04 & $0 \cdot 4$ & $0 \cdot 122$ \\
\hline Other milk/cream & $0 \cdot 19$ & 0.03 & $14 \cdot 2$ & 0.21 & 0.02 & $16 \cdot 2$ & 0.25 & 0.02 & $18 \cdot 4$ & 0.019 \\
\hline Yoghurt/fromage frais & $3 \cdot 60$ & $0 \cdot 21$ & 38.9 & $4 \cdot 26$ & $0 \cdot 16$ & $47 \cdot 4$ & $4 \cdot 27^{\star}$ & $0 \cdot 14$ & $48 \cdot 6$ & $<0.001$ \\
\hline Eggs & 0.97 & 0.07 & $26 \cdot 6$ & 1.03 & 0.06 & $26 \cdot 5$ & $1 \cdot 20^{*}$ & 0.05 & $31 \cdot 8$ & 0.001 \\
\hline Cheese & $1 \cdot 40$ & 0.07 & $49 \cdot 5$ & 1.54 & 0.06 & $55 \cdot 4$ & $1 \cdot 76^{\star}$ & 0.05 & $61 \cdot 4$ & $<0.001$ \\
\hline
\end{tabular}


fat/sugar-containing foods such as biscuits, cakes, puddings, chocolates and sweets but very similar levels of meat and meat products. Other studies have shown similar relationships with fatty/sugary foods and with meat products ${ }^{(37,44)}$. Perhaps it was less easy to forget to record meats usually eaten as part of a meal than biscuits or sweets often eaten as snacks, or it could be that the snack foods which have a reputation as being unhealthy were selectively omitted by the under-reporters.

Comparisons made between the intakes in these children and nutrient recommendations showed that nutrient intakes were adequate; however, fat and saturated fat intakes were much higher than the recommended levels, both exceeding the recommendation by more than $10 \%$. Eating a high-fat diet has been associated with heart disease and cancer risk ${ }^{(45)}$. Average NSP intake was much lower than current health guidelines recommend and the mean intake of NMES was much higher, almost double the recommended maximum.

In assessing the relationship between child diet and maternal education only those reporting plausible energy intakes were used, this was in order to avoid bias from differential under-reporting. Furthermore we found that there was no independent association between misreporting status and maternal education. There was no evidence of a gradient in energy intake between maternal education groups and differences in macronutrient intakes were small. However, total sugars intake increased with education while NMES intake was similar, suggesting that the higher education group consumed more intrinsic sugars. This was probably explained by their higher consumption of fruit.

Substantial differences were seen in types of food and drinks consumed between maternal education groups. Intakes of fruit, fruit juice, raw vegetables and nuts were more than a third higher in the highest education group compared with the lowest. This probably explained their higher vitamin $\mathrm{C}$ intake. A diet rich in fruit and vegetables is thought to be beneficial to health ${ }^{(46)}$ and a recent review of studies in adults has shown that fruit and vegetable consumption was higher in those with better education $^{(47)}$. Previous studies in adolescents found a relationship between educational level of the household and raw vegetable intake ${ }^{(48)}$, and low intakes of fruit and high intakes of sweetened beverages were found in children from households of low educational attainment ${ }^{(49)}$. In the present study, sweetened drinks intake was similar across the education groups, but diet drinks intake was considerably higher in the low maternal education group. In line with the higher NSP intake in the highest education group, high-fibre breakfast cereals and brown and wholemeal bread were more likely to be consumed by them. A balanced diet should include a sufficient amount of fibre to promote long-term health ${ }^{(45)}$.

In contrast, a trend towards higher intakes of less healthy foods was shown in the lowest education group. 
Intakes of various types of processed meat were 15-40\% higher in the low compared with the high education group. Intakes of both chocolate confectionery and savoury snacks were 10-25\% higher in the lowest education group; however, there was little difference in consumption levels for sugar confectionery and all these foods were consumed by large numbers of children in all groups. Other studies have shown that consumption of snack foods was more prominent in children with low $v$. higher educated mothers and that the converse is true with regard to eating fruit and vegetables ${ }^{(15,50)}$. Furthermore, consumption of savoury snacks will add to the high non-discretionary $\mathrm{Na}$ intakes found in the current sample, which fell slightly with increased maternal education status. High $\mathrm{Na} /$ salt intakes are associated with increased risk of hypertension and strokes in later life ${ }^{(43)}$.

Differences in diet quality according to maternal education level were evident in the present study. Various studies in adults have shown that those with a high educational status tend to consume a better-quality $\operatorname{diet}^{(51,52)}$. Inequality in family background has been shown to impact on children's diets; for example, a study of 2149 American girls aged 9-10 years found on average that those whose parents had a higher educational status had a healthier diet ${ }^{(53)}$.

There are several limitations to the present study. It was conducted in one geographical area of England and so may not be applicable throughout the UK; however, the cohort was reasonably representative of the UK population at recruitment and we have shown that the dietary intakes in the study were comparable with those of a nationally representative cross-sectional sample, NDNS. Under-reporting of energy intake occurred in approximately $36 \%$ of subjects in the current sample; again this was comparable with NDNS. The assessment to classify plausibility of intake was limited because objective physical activity was not measured at 10 years, thus necessitating the assumption of a moderate physical activity level for everyone. This is likely to have led to bias ${ }^{(54)}$. However, the misreporting level was fairly reasonable which may in part be due to parents helping their 10-year-olds to complete the diet diaries and the fact that children were interviewed with their diaries to fill in any gaps. The interviewers obtained $24 \mathrm{~h}$ recalls of the diet from the previous day if no diary was produced, thus maximizing the number of participants. A maximum of $3 \mathrm{~d}$ of diet recording was obtained from most children in the present study, whereas $7 \mathrm{~d}$ of recording is likely to provide a more reliable estimate of intake. We considered that asking for more recording days would put unnecessary pressure on the children and their parents, which could have impacted adversely on response rates. One of the main strengths of the study is its large sample size and high participation rate. However those from higher educational groups were more likely to attend the 10-year clinic and provide dietary data and the comparisons between education groups were limited to plausible reporters of energy, thus reducing the number of subjects further. This will have had an impact on the results such that the observed social gradients were likely to appear smaller than in reality, as the less educated who stayed in the study may be different in ways that make their diet better than those who dropped out.

In conclusion, we have shown that maternal education level is related to differences in children's diet at 10 years. Lower maternal education level is associated with less healthy food choices that could have a detrimental effect on later health outcomes. Further research is needed to establish whether these associations are explained by related socio-economic factors such as family income and parental occupation or are independent of these factors. Little is known about the mechanisms that underlie the differences in eating behaviours according to maternal educational status, although some important clues have been obtained by qualitative methods ${ }^{(54)}$. For example, women of lower educational attainment perceived themselves to have a lack of control over food choice for their families compared with better educated women ${ }^{(55)}$.

Further work should assess how socio-economic indicators relate to food choice. Clarifying associations between educational level, socio-economic status and nutrition may help in the development of interventions to improve diet quality, especially in the poorly educated, thus helping to reduce health inequalities.

\section{Acknowledgements}

The UK Medical Research Council, the Wellcome Trust and the University of Bristol provide core support for ALSPAC. The work of P.M.E. was supported by the Arthritic Association. The World Cancer Research Fund International and the Wellcome Trust funded the research reported herein. The authors declare no conflict of interest. V.L.C. drafted the manuscript, performed statistical analysis and was involved in interpretation. I.S.R., A.R.N., L.R.J. and P.M.E. contributed to the collection and interpretation of the data. This publication is the work of the authors and V.L.C. and P.M.E. are guarantors for the contents of this paper. The authors are extremely grateful to all the families who took part in this study, the midwives for their help in recruiting them and the whole ALSPAC team, which includes interviewers, computer and laboratory technicians, clerical workers, research scientists, volunteers, managers, receptionists and nurses.

\section{References}

1. Buttriss J (2002) Nutrition, health and schoolchildren. Nutr Bull 27, 275-316.

2. Variyam JN, Blaylock J, Lin B et al. (1999) Mother's nutrition, knowledge and children's dietary intakes. Am J Agric Econ 81, 373-384. 
3. Parliamentary Office of Science and Technology (2003) Improving Children's Diet. London: The Parliamentary Office of Science and Technology.

4. Vereecken C \& Maes L (2000) Eating habits, dental care and dieting. In Health and Health Behaviour Among Young People. A WHO Cross-National Study (HBSC) International Report, pp. 89-93 [C Currie, K Hurrelmann, W Settertobulte et al., editors]. Copenhagen: WHO Regional Office for Europe.

5. Feinstein L, Sabates R, Sorhaindo A et al. (2008) Dietary patterns related to attainment in school: the importance of early eating patterns. J Epidemiol Community Health 62 , 734-739.

6. Rogers I, Emmett P \& the ALSPAC Study Team (2003) The effect of maternal smoking status, educational level and age on food and nutrient intakes in preschool children: results from the Avon Longitudinal Study of Parents and Children. Eur J Clin Nutr 57, 854-864.

7. Wachs TD, Creed-Kanashiro H, Cueto S et al. (2005) Maternal education and intelligence predict offspring diet and nutritional status. J Nutr 135, 2179-2186.

8. Story M, Neumark-Sztainer D \& French S (2002) Individual and environmental influences on adolescent eating behaviours. J Am Diet Assoc 102, 40-51.

9. Wardle J (1995) Parental influences on children's diet. Proc Nutr Soc 54, 747-758.

10. Hanson NI, Neumark-Sztainer D, Eisenberg ME et al. (2004) Associations between parental report of the home food environment and adolescent intakes of fruit, vegetables and dairy foods. Public Health Nutr 8, 77-85.

11. St. John Alderson T \& Ogden J (1999) What do mothers feed their children and why? Health Educ Res 14, 717-727.

12. Haapalahti M, Mykkanen H, Tikkanen S et al. (2002) Meal patterns and food use in 10- to 11-year-old Finnish children. Public Health Nutr 6, 365-370.

13. Klesges RC, Stein RJ, Eck LH et al. (1991) Parental influences on food selection in young children and its relationship to childhood obesity. Am J Clin Nutr 53, 859-864.

14. Wachs TD \& McCabe G (2001) Relation of maternal intelligence and schooling to offspring nutritional intake. Int J Behav Dev 25, 444-449.

15. Aranceta J, Perez-Rodrigo C, Ribas L et al. (2003) Sociodemographic and lifestyle determinants of food patterns in Spanish children and adolescents: the enKid study. Eur J Clin Nutr 57, Suppl. 1, S40-S44.

16. Vereecken C \& Maes E (2004) Influence of mother's educational level on food parenting practices and food habits of young children. Appetite 43, 93-103.

17. De Irala-Estevez J, Groth M, Johansson L et al. (2000) A systematic review of socio-economic differences in food habits in Europe: consumption of fruit and vegetables. Eur J Clin Nutr 54, 706-714.

18. Lv N \& Cason K (2004) Dietary pattern change and acculturation of Chinese Americans in Pennsylvania. J Am Diet Assoc 104, 771-778.

19. Golding J, Pembrey M, Jones R et al. (2001) ALSPAC - The Avon Longitudinal Study of Parents and Children I. Study methodology. Paediatr Perinat Epidemiol 15, 74-87.

20. Price G, Paul A, Key F et al. (1995) Measurement of diet in a large national survey: comparison of computerised and manual coding of records in household measures. J Hum Nutr Diet 8, 417-428.

21. Gregory J \& Lowe S (2000) National Diet and Nutrition Survey: Young People Aged 4 to 18 Years. vol. 1: Report of the Diet and Nutrition Survey. London: The Stationery Office.

22. Wreiden W, Longbottom P, Adamson A et al. (2008) Estimation of typical food portion sizes for children of different ages in Great Britain. Br J Nutr 99, 1344-1353.
23. Holland B, Welch A, Unwin I et al. (1991) McCance E Widdowson's The Composition of Foods, 5th ed. Cambridge: Royal Society of Chemistry.

24. Holland B, Unwin I \& Buss D (1988) Cereals and Cereal Products. Third Supplement to 4 th Edition of McCance $\mathcal{E}$ Widdowson's The Composition of Foods. Cambridge: Royal Society of Chemistry.

25. Holland B, Unwin I \& Buss D (1989) Milk Products and Eggs. Fourth Supplement to 4th Edition of McCance $\&$ Widdowson's The Composition of Foods. Cambridge: Royal Society of Chemistry.

26. Holland B, Unwin I \& Buss D (1991) Vegetables, Herbs and Spices. Fifth Supplement to 4 th Edition of McCance \& Widdowson's The Composition of Foods. Cambridge: Royal Society of Chemistry.

27. Holland B, Welsh A \& Buss D (1992) Fruits and Nuts. First Supplement to 5th Edition of McCance \& Widdowson's The Composition of Foods. Cambridge: Royal Society of Chemistry.

28. Holland B, Welsh A \& Buss D (1992) Vegetable Dishes. Second Supplement to 5th Edition of McCance E Widdowson's The Composition of Foods. Cambridge: Royal Society of Chemistry.

29. Holland B, Brown J \& Buss D (1993) Fish and Fish Products. Third Supplement to 5th Edition of McCance $\mathcal{E}$ Widdowson's The Composition of Foods. Cambridge: Royal Society of Chemistry.

30. Chan W, Brown J \& Buss D (1994) Miscellaneous Foods. Fourth Supplement to 5th Edition of McCance \& Widdowson's The Composition of Foods. Cambridge: Royal Society of Chemistry.

31. Chan W, Brown J, Lee S et al. (1995) Meat, Poultry and Game. Fifth Supplement to 5th Edition of McCance \& Widdowson's The Composition of Foods. Cambridge: Royal Society of Chemistry.

32. Department of Health (1991) Dietary Reference Values of Food Energy and Nutrients for the UK. Report of the Committee on Medical Aspects of Food Policy. Report on Health and Social Subjects no 41. London: HMSO.

33. Cole TJ, Bellizzi MC, Flegal KM et al. (2000) Establishing a standard definition for child overweight and obesity worldwide: international survey. BMJ 320, 1240-1243.

34. Cole TJ, Flegal KM, Nicholls D et al. (2007) Body mass index cut offs to define thinness in children and adolescents: international survey. BMJ 335, 194-201.

35. Willett W \& Stampfer MJ (1998) Implications of total energy intake for epidemiological analysis. In Nutritional Epidemiology, pp. 273-297 [W Willett, editor]. New York: Oxford University Press.

36. Westerterp KR \& Goris AHC (2002) Validity of the assessment of dietary intake: problems of misreporting. Curr Opin Clin Nutr Metab Care 5, 489-493.

37. Livingstone MBE \& Black AE (2003) Markers of the validity of reported energy intake. J Nutr 133, Suppl. 3, 895S-920S.

38. Livingstone MBE, Robson PJ \& Wallace JMW (2004) Issues in dietary intake assessment of children and adolescents. Br J Nutr 92, Suppl. 2, S213-S222.

39. Rennie KL, Coward A \& Jebb SA (2007) Estimating underreporting of energy intake in dietary surveys using an individualised method. Br J Nutr 97, 1169-1176.

40. Torun B (2005) Energy requirements of children and adolescents. Public Health Nutr 8, 968-993.

41. Glynn L, Emmett P, Rogers I et al. (2005) Food and nutrient intakes of a population sample of 7-year-old children in the south-west of England in 1999/2000 - what differences does gender make? J Hum Nutr Diet 18, 7-19.

42. Scientific Advisory Committee on Nutrition (2003) Salt and Health. London: The Stationery Office. 
43. Riddoch C, Mattocks C, Deere K et al. (2007) Objective measurement of levels and patterns of physical activity. Arch Dis Child 92, 963-969.

44. Bingham SA, Cassidy A, Cole TJ et al. (1995) Validation of weighed records and other methods of dietary assessment using the $24 \mathrm{~h}$ urine nitrogen technique and other biological markers. Br J Nutr 73, 531-550.

45. World Health Organization/Food and Agriculture Organization of the United Nations (2003) Diet, Nutrition and the Prevention of Chronic Diseases. Report of a Joint WHO/FAO Expert Consultation. WHO Technical Report Series no. 916. Geneva: WHO.

46. Steffen LM (2006) Eat your fruit and vegetables. Lancet 367, 278-279.

47. Roos G, Johansson L, Kasmel A et al. (2000) Disparities in vegetable and fruit consumption: European cases from the north to the south. Public Health Nutr 4, 35-43.

48. Roos EB, Hirvonen T, Mikkila V et al. (2001) Household educational level as a determinant of consumption of raw vegetables among male and female adolescents. Prev Med 33, 282-291.

49. Cullen K, Ash D, Warneke C et al. (2002) Intakes of soft drinks, fruit, flavoured beverages and fruit and vegetables by children in grades 4 through 6. Am J Public Health $\mathbf{9 2}$, 1475-1478.

50. Brekke HK, van Odijk J \& Ludivigsson J (2007) Predictors and dietary consequences of frequent intake of high sugar, low nutrient foods in 1-year-old children participating in the ABIS study. Br J Nutr 97, 176-181.

51. Robinson SM, Crozier SR, Borland SE et al. (2004) Impact of educational attainment on the quality of young women's diets. Eur J Clin Nutr 58, 1174-1180.

52. Darmon N \& Drewnowski S (2008) Does social class predict diet quality? Am J Clin Nutr 87, 1107-1117.

53. Crawford PB, Obarzanek E, Schreiber GB et al. (1995) The effects of race, household income and parental education on nutrient intakes of 9- and 10-year-old girls. NHLBI Growth and Health Study. Ann Epidemiol 5, 360-368.

54. Black AE (2000) Critical evaluation of energy intake using the Goldberg cut-off for energy intake:basal metabolic rate. A practical guide to its calculation, use and limitations. Int J Obes Relat Metab Disord 24, 1119-1130.

55. Barker M, Lawrence WT, Skinner TC et al. (2008) Constraints on food choices of women in the UK with lower educational attainment. Public Health Nutr 11, 1229-1237. 